\title{
PERCEPCIÓN DE LA EPILEPSIA POR GRUPOS INDÍGENAS: CONCEPTUALIZACIONES CLÍNICAS
}

\author{
Eduardo Palacios S.MD*, Leonardo Hernández MD**, Rosana Solano Peláez MD***
}

\section{Resumen}

La epilepsia cursa con altas tasas de incidencia y prevalencia; hay más de 50 millones de personas diagnosticadas a nivel mundial. Desde los evangelios y durante la edad media y el renacimiento, predominaron los tratamientos mágicos y religiosos ante crisis epilépticas; durante el siglo $\mathrm{XX}$, algunas poblaciones indígenas siguieron manejando esta enfermedad con rituales. Objetivo: revisión ampliada de la literatura sobre la percepción de los indígenas acerca de la epilepsia y sus manifestaciones clínicas. Métodos: revisión documental ampliada de estudios cualitativos y de revisión de tema, publicados entre 1977 y 2013. Resultados: de 73 publicaciones, 50 cumplieron con los criterios de selección, de estas 22 eran revisiones del tema, 11 estudios cualitativos y descriptivos, y 17 documentos conformados por libros, memorias de congresos, monografías y documentos de la Organización Mundial de la Salud seleccionados de las citas bibliográficas de los artículos escogidos. Se encontró que las tribus indígenas en África piensan que la epilepsia se origina en el mal de ojo, hechizo o brujería; en Asia la medicina tradicional china la atribuye a procesos físicos que bloquean el sistema cardíaco y en la India el ayurveda a eventos psicofisiológicos. En América (sur y centro) los incas peruanos hacen una buena descripción en cuanto al origen, clínica y diagnóstico, en Bolivia los chipayas identifican factores desencadenantes de crisis y establecen un pronóstico, aunque la etiología la atribuyeron a un mal espíritu. Los mayas en Méjico y los kamayurá en Brasil le dieron una connotación mágico-religiosa. Conclusiones: se encontró una fuerte concepción mágica en todas las tribus del territorio africano, mientras en América hay diferencias entre los grupos, unos con concepciones de origen orgánico como los incas del Perú y otras culturas como maya, uru-chipaya y kamayurá con percepción mágico-religiosa.

Palabras clave: indígenas, epilepsia, etnología.

\section{PERCEPTIONS ABOUT EPILEPSY OF INDIGENOUS GROUPS: CLINICAL CONCEPTUALIZATIONS}

\section{Abstract}

Epilepsy has high rates of incidence and prevalence. Worldwide there are more than $\mathbf{5 0}$ million people diagnosed with this condition. From the gospels to the middle ages and the renaissance, magic and religious treatments were predominant for epileptic crises. During the 20th century, some indigenous populations have continued managing this disease through rituals. Objective: an extensive review of literature on the perception of indigenous people about epilepsy and its clinical manifestations. Methods: broad documental review of qualitative assays and topic reviews, published between 1977 and 2013. Results: of 73 publications, 50 met the selection criteria, and of these 22 were reviews of this topic, 11 were descriptive qualitative studies and 17 were documents conformed by books, congress memories, monographs and World Health Organization documents selected of the bibliographic cites of chosen articles. It was found that African indigenous tribes believe epilepsy is due to an evil eye spell, possession or bewitchment; in Asia,

Fecha recibido: junio 26 de 2013 - Fecha aceptado: septiembre 5 de 2013

* Jefe del Servicio de Neurología del Hospital de San José. Miembro de la Sociedad de Cirugía de Bogotá. Profesor Titular de Neurología, Fundación Universitaria de Ciencias de la Salud. Bogotá DC, Colombia.

\footnotetext{
** Coordinador encargado del Servicio de Neurología, Hospital de San José. Instructor Asistente, Fundación Universitaria de Ciencias de la Salud, Bogotá DC, Colombia.

*** Residente IV de Neurología, Fundación Universitaria de Ciencias de la Salud, Bogotá DC, Colombia.
} 
Chinese traditional medicine attributes the cause to physical processes which block the cardiac system; Ayurveda to physiological and psychological events and in America (South and Central) the Peruvian Incas make a good description regarding origin, clinical features and diagnosis; in Bolivia the Chipayas identify triggering factors of crises and establish a prognosis, although they attribute etiology to an evil spirit. The Mayas in Mexico and the Kamayurá in Brazil gave epilepsy a magical-religious connotation. Conclusions: a strong magical conception was found in all the tribes of the African territory, while there are differences between groups in America, some with organic origin conceptions such as the Incas from Peru and other such as the Maya, Uru-Chipaya and Kamayurá cultures which have a magical-religious perception.

Key words: indigenous population, epilepsy, ethnology.

\section{Introducción}

La epilepsia es una de las principales enfermedades neurológicas con altas tasas de prevalencia e incidencia y constituye un importante problema de salud pública. ${ }^{1}$ Se estima que el $80 \%$ de los 50 millones de personas con epilepsia en todo el mundo, viven en países en desarrollo. ${ }^{2,3}$ Es también una de las condiciones reconocidas más antiguas del mundo, históricamente atribuida a la posesión sagrada o demoníaca, un castigo divino, brujería o de contagio, y se asocia con el miedo, la vergüenza, la culpa y el misticismo., En muchas sociedades se entiende que es crónica e incurable, pero controlable. $^{6-8}$

El término epilepsia procede del griego "epilambanein" y significa ser sobrecogido o poseído bruscamente por fuerzas exteriores, expresando la concepción animista que desde tiempos inmemoriales asoció esta enfermedad con fuerzas sobrenaturales y gestó uno de los estigmas fijados con más solidez en el inconsciente colectivo de la humanidad. ${ }^{9}$

Hipócrates intentó refutar la conexión entre la epilepsia y lo divino argumentando contra las creencias acerca de los poderes místicos atribuidos a las personas con esta enfermedad y la causalidad divina del trastorno. Sin embargo, a lo largo de la historia de la humanidad diferentes informes anecdóticos asociaron el tratamiento de la enfermedad con elementos religiosos. Así, desde los evangelios, en la edad media y el renacimiento, predominaron los tratamientos mágicos y religiosos para las personas que sufrían de crisis epilépticas; y durante el siglo XX se continuó asociando el manejo religioso para este mal. ${ }^{10}$
La comprensión acerca de la conceptualización de la enfermedad está limitada por falta de pruebas documentales, así como también por la renuencia de algunos pacientes y gente del común a admitir sus creencias verdaderas. En comunidades subdesarrolladas, se ha visto una fuerte aceptación de lo sobrenatural como una de las causas de la epilepsia ${ }^{11}$, hipótesis corroborada por algunos estudios: el Shona (Gelfland, 1973) ${ }^{12}$ puede verse como la venganza de los antepasados agraviados, el yoruba en Nigeria (Osuntoku, 1977) ${ }^{13}$ es el signo de la visita del diablo y muchos pacientes en un estudio en Iraq (Hamdi y col.1977) $)^{14}$, consideran la enfermedad debida a "los poderes, el fantasma, o el mal de ojo." En la India (Virmani y col.1977) ${ }^{15}$, el $6 \%$ de los enfermos más pobres y no menos del $35 \%$ de sus familiares la consideran debida a causas sobrenaturales (no definidas). En Ecuador (Estrella, 1977) ${ }^{16}$ ciertos casos creen que tienen causa sobrenatural. De hecho, las etiologías naturales las aceptan algunas comunidades, muchas veces junto con las sobrenaturales. En Kenya, por ejemplo, algunos que han sufrido accidentes reconocen que el "golpe en la cabeza" puede ser una de las razones de su enfermedad y también que la herencia puede ser un factor causal natural. Las huellas de esta creencia permanecen en muchos lugares, como Madagascar, Tanzania (Jilek-Aall y col.1979) ${ }^{17}$ y Nigeria (Arawatife y col. 1985). ${ }^{18}$ En el caso particular de los pueblos indígenas americanos, afirman Carod y Vázquez (2007) que la epilepsia no se disoció de una conceptualización relacionada con creencias religiosas. Sin embargo, existen escasos estudios sobre el tema, así como de los conocimientos, las actitudes y las prácticas sobre este padecimiento. ${ }^{19} \mathrm{~A}$ continuación se presenta una revisión sobre la percepción que 
poseen los indígenas de la epilepsia y sus manifestaciones clínicas en África y América.

\section{Métodos}

Criterios de elegibilidad: estudios cualitativos y de revisión de tema publicados desde 1977 hasta 2013, que hicieran relación con cualquier grupo de edad; solo se tomaron en cuenta artículos en español e inglés. Así mismo se buscaron investigaciones acerca de los conceptos clínicos que poseen los grupos indígenas. Fuentes de información: la búsqueda se realizó en Medline, Lilacs, Google Académico $y$ citaciones bibliográficas de artículos escogidos, revisando los documentos allí referenciados. Los términos utilizados fueron: en Medline (PubMed): "Epilepsy"[Mesh] AND "Indians, South American"[Mesh], epilepsy indigenous, epilepsy Indians. Lilacs: anthropological and epilepsy, traditional and epilepsy. Google: indigenous and epilepsy.

Selección de los estudios: se revisaron los títulos y resúmenes de los artículos escogiendo los que cumplían con los criterios de elegibilidad, así como las referencias de los artículos escogidos y con base en el titulo se tomaron los elegibles. Después se procedió a su búsqueda y revisión y se seleccionaron los que reunían las condiciones requeridas. En cada artículo se identificó la información del autor, año de publicación, población, tipo de estudio y conclusiones. Según la legislación Colombiana esta investigación se considera un estudio sin riesgo por lo cual no requirió de valoración por el comité de ética institucional.

\section{Resultados}

En la búsqueda realizada con los términos descritos se identificaron 111 títulos, de estos se revisaron los resúmenes de 73 por ser sugestivos de contener información relacionada con la percepción que poseen los indígenas acerca de la epilepsia y sus manifestaciones clínicas. Posterior a esta revisión se escogieron 33 artículos por cumplir con los criterios de selección. De estos, 22 eran de revisión y 11 estudios que tres eran descriptivos y ocho cualitativos (Tabla 1). También se revisaron las citas bibliográficas de dichos artículos escogiendo 17 documentos conformados por libros, memorias de congresos, monografías y documentos de la Organización Mundial de la Salud, cuyos resultados se analizan a continuación.

\begin{tabular}{|c|c|c|c|c|}
\hline Autor & Título & Revista & Año & Tipo de estudio \\
\hline Caveness W & $\begin{array}{l}\text { Survey of public attitudes towards epilepsy in } 1979 \text { with an } \\
\text { indication of trends over the last thirty years }\end{array}$ & Epilepsia & 1980 & Descriptivo \\
\hline Jacoby $A$ & $\begin{array}{l}\text { Meanings of epilepsy in its sociocultural context and implica- } \\
\text { tions for stigma: findings from ethnographic studies in local } \\
\text { communities in China andVietnam }\end{array}$ & Epilepsy \& Behavior & 2000 & Cualitativo \\
\hline Jacoby $A$ & $\begin{array}{l}\text { Meanings of epilepsy in its sociocultural context and implica- } \\
\text { tions for stigma: findings from ethnographic studies in local } \\
\text { communities in China andVietnam }\end{array}$ & Epilepsy \& Behavior & 2008 & Cualitativo \\
\hline Mushi a D & $\begin{array}{l}\text { Social-cultural aspects of epilepsy in Kilimanjaro Region, } \\
\text { Tanzania: Knowledge and experience among patients and } \\
\text { carers }\end{array}$ & Epilepsy \& Behavior & 2011 & Cualitativo \\
\hline Jilek-Aall L & $\begin{array}{l}\text { Clinical and genetic aspects of seizure disorders prevalent in } \\
\text { an isolated African population }\end{array}$ & Epilepsia & 1979 & Descriptivo \\
\hline Arawatife A & Epilepsy and psychosis: a comparison of societal attitudes & Epilepsia & 1985 & Cualitativo \\
\hline Carod F & $\begin{array}{l}\text { An Anthropological Study about Epilepsy in Native Tribes } \\
\text { from Central and South America }\end{array}$ & Epilepsia & 2007 & Cualitativo \\
\hline Carod a F & $\begin{array}{l}\text { Pensamiento mágico y epilepsia en la medicina tradicional } \\
\text { indígena }\end{array}$ & Rev Neurol & 1998 & Cualitativo \\
\hline Carod-Artal FJ & $\begin{array}{l}\text { Estudio etnográfico sobre las enfermedades neurológicas y } \\
\text { mentales entre los urus-chipayas del altiplano andino }\end{array}$ & Rev Neurol & 2005 & Cualitativo \\
\hline Millogo A & $\begin{array}{l}\text { Epilepsy and traditional medicine in Bobo-Dioulasso (Bur- } \\
\text { kina Faso) }\end{array}$ & $\begin{array}{l}\text { Acta Neurologica } \\
\text { Scandinavica }\end{array}$ & 2004 & Descriptivo \\
\hline Baskind R & Epilepsy care in Zambia: a study of traditional healers & Epilepsia & 2005 & Cualitativo \\
\hline
\end{tabular}




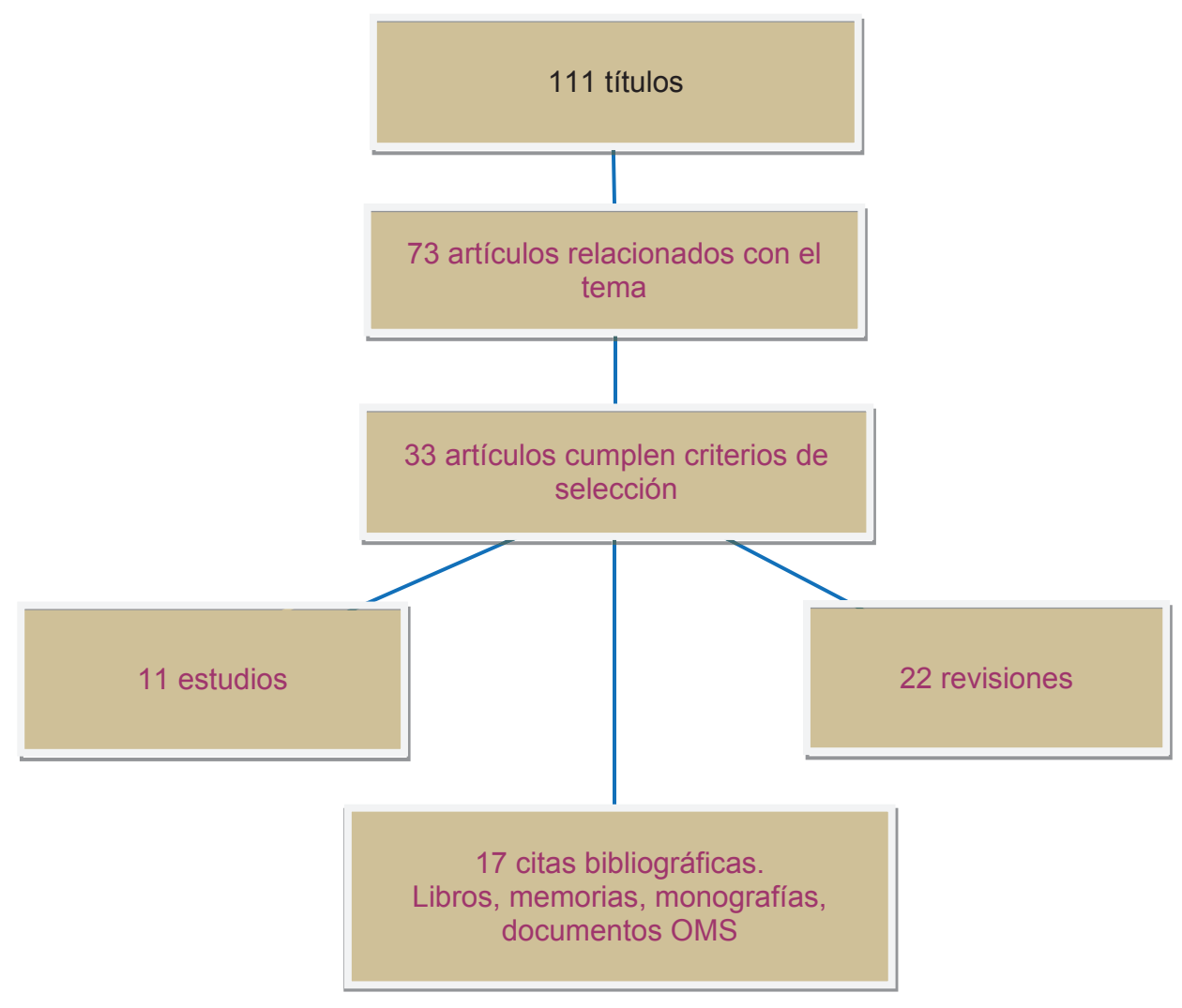

Figura I. Selección de artículos.

\section{África}

Noroeste de Camerun y sur de Nigeria: en estas zonas habitan las etnias bassá, fufulve y bambiliké para quienes el origen de la epilepsia se basa en un pensamiento mágico, siendo el mal de ojo, el hechizo y la brujería la fuente de la enfermedad. ${ }^{20}$ En África central y subsahariana se conoce como "kin doki" y es producida por el mal de ojo o hechizo, el cual puede ser emitido por un enemigo o familiar enemistado, generando enfermedad y convulsiones. Para prevenirlas los curanderos y los iniciados realizan cortes en la cara de los afectados creando cicatrices, para que las malas influencias se fijen en estas y no en los ojos, evitando así el mal de ojo. En la Nigeria rural se utiliza un tratamiento tradicional para las crisis febriles, el cual ha caído en desuso en las últimas décadas. Consiste en tomar una infusión de diversas plantas mezcladas con orina de vaca diluida en licor fermentado de maíz, algunos niños que la han recibido han presentado intoxicación por la acción de la nicotina del tabaco mezclada con el alcohol.
Sur de Tanzania: allí tiene su asentamiento la etnia wangoni ${ }^{21}$ en donde la epilepsia y convulsión van ligadas al mal de ojo, producido por alguien cercano en la comunidad, a veces incluso de modo inconsciente. Bajo el mal de ojo la persona perderá el sentido, caerá al suelo, emitirá espuma y se volverá rígida. En swahilli este hecho se denomina 'kifafa' que correspondería a nuestro término crisis o convulsión. Ante esta situación, el brujo de la tribu, 'mganga' en swahilli, y la familia del afectado deben reunirse para establecer quién es el causante de ese maleficio. Una vez identificado se procede a realizar el ritual de curación tanto al epiléptico como al causante del mal. Este último debe someterse a la depilación completa del cuerpo, si no lo acata es desterrado y el epiléptico es aceptado pero con los estigmas que conlleva la enfermedad. ${ }^{22}$ Entre los wagoporos, también en Tanzania, creen que la epilepsia tiene afinidad por la luna, algunos asistentes al Mahenge Clinic for Epilepsy refieren que la mayoría de las crisis suceden en la fase de luna llena, mientras otros las refieren en la luna nueva. En 1994 se llevó a cabo un estudio en el área de influencia de Mahenge y 
en Ruha una zona remota donde no se ha establecido clínica de epilepsia, que presenten alta prevalencia de la afección. En Ruha la mayoría de los enfermos se veían tímidos, deprimidos y malhumorados, mientras los que recibían tratamiento en la clínica de epilepsia Mahenge permanecían alegres y bien integrados a su vida familiar normal. ${ }^{23}$

\section{América}

Paraguay: en la región del Chaco paraguayo, Bolivia y la provincia de Misiones en Argentina ${ }^{24}$ los guaraníes son conocedores de una amplia gama de "yuyos" plantas medicinales que aplican a una gran variedad de patologías.

La epilepsia se considera una entidad mágico-religiosa y es concebida como un desequilibrio entre el alma sagrada y el alma animal presentes en cada individuo, casi siempre provocado por alguna influencia maléfica de algún enemigo externo a la comunidad o de un pariente enemistado. El "paye" o chamán realiza un rito en el que produce un trance con alucinógenos y establece la naturaleza de la enfermedad y cómo hay que curarla. El tratamiento se basa en la ingesta de un preparado nativo que incluye la raíz de 'caaré' (Chenopodium ambrosioides, nombre vulgar, té de los jesuitas), hojas de 'curaturá' (Fagara hiemalis, nombre vulgar, naranjillo) y hojas de 'ysaú caá' (Poiretia tetraphylla, nombre vulgar, té de la pampa), que se administra en forma indefinida y se complementa con una dieta rica en sopa de verduras, huevos, dulce de calabaza y frutas en general. Los condimentos y bebidas alcohólicas se consideran alimentos prohibidos. ${ }^{22}$

Perú: sonko nanay o enfermedad del corazón representa en lengua quechua (usada por los incas) la descripción de un evento epiléptico. Sonko tiene diferentes significados: corazón, mente, memoria y algunas veces cerebro. Es el axis o centro del cuerpo humano y al mismo tiempo de la mente, colocado en un área difusa en la región precordial. Nanay significa dolor o enfermedad. Sonko nanay se utilizó para describir eventos epilépticos y síncopes. En los escritos de Garcilaso de la Vega "comentarios reales de los incas" él describió eventos epilépticos focales "cuando hay espasmos de los ojos o un zumbido en los oídos o sacudidas en otras partes del cuerpo, puede ser difícil escuchar o ver del lado involucrado".

Sal y Rozas investigó sobre la epilepsia en el Perú antiguo y encontró que entre los indios nativos sonko nanay podría causar la muerte, es posible que hacían referencia a la hoy conocida como muerte súbita en epilepsia. Para esta fue usado el término huañuyonkay (enfermedad de la muerte). Los incas sabían que la epilepsia se podía presentar de diferentes maneras. Describieron los síntomas con diferentes palabras: songo-piti y songo-chiriray, para gran mal, nahuin-ampin para el petit mal, upayacurin como arresto del comportamiento y upakundiya el engaño volcánico. La causa de la enfermedad se explicaba a través de mitos o narraciones místicas en las cuales la epilepsia era el resultado de castigos divinos. Eran los mitos de Llaqui y Aya Huayra. Llaqui significa tristeza en quechua, que afecta el sonko después de una tragedia personal como la pérdida de un ser querido y el sonko enferma. Aya significa cadáver y huayra viento, este último es la venganza o castigo de los ancestros muertos por el mal comportamiento y puede castigar el sonko. ${ }^{26}$

El diagnóstico se basa en el sentido clínico y la observación de hampicamayoc (médico curandero), pero en ocasiones requiere métodos mágicos, el más sistematizado es el de cuy (cobaya) que al pasarse por el cuerpo del enfermo permite reconocer dónde está la enfermedad. Las medicinas para la curación son producto de los tres reinos sin probada actividad antiepiléptica y significado probablemente simbólico y mágico. Sonko-rumi (piedra del corazón) actúa con nivel curativo sobre sonko-nanay (enfermedad del corazón). También se empleó el polvo de los picapedreros; rumipa shonkon (granito) y pasca (piedra caliza). En los derivados del reino animal se usó carne o sangre de cóndor, llama, golondrina, zorzal, puma y perro. Entre las plantas tuvieron preferencia las odoríferas que incluye el toronjil, pimpinela, alhelí, maqué, ruda y también huayroro que son pepitas de dos colores. 
En cuanto a la cirugía practicada por sircay (cirujano) se realizaban dos tipos: la ficticia y la real. Ambas han sido descritas en innumerables culturas. La ficticia consiste en efectuar una herida superficial en el área o zona afectada a fin de que salga el espíritu o el humor causante del mal. Dentro del campo de cirugía real está la trepanación del cráneo que plantea incógnitas básicas ¿Cuál era el propósito de la trepanación? ¿Tuvo indicaciones precisas? ¿Fue acaso parte de un hecho ritual o mágico? ¿Fue en esencia un elemento terapéutico? Es imposible dar respuestas integradas a estos interrogantes. Requieren un juicio equilibrado y una discusión objetiva. El análisis de los cráneos ha sido efectuado por varios médicos historiadores peruanos. Aquí sólo mencionaremos a F. Cabieses E. Fernández y J. Lastres. ${ }^{27}$ Podemos ver con claridad la posibilidad de que hayan efectuado estas trepanaciones en casos de traumas en la cabeza cerrados o abiertos y en pacientes que sufrían de dolor de cabeza. Además, se piensa que lo más probable es que pudieran haber sido efectuadas en enfermos mentales y también para la epilepsia. Lastres afirma que la observación penetrante del indígena le hizo llegar al convencimiento de que numerosos casos de fractura conminutiva de los huesos frontales o frontoparietales traían a la larga síntomas de convulsión y es justo relacionar el traumatismo con el sistema nervioso. Por ello, la gran mayoría de trepanaciones asientan en los compartimentos anteriores del cráneo. El demonio-enfermedad localizado en los fragmentos de la fractura era lo que determinaba la convulsión o la 'ausencia momentánea del ánima'. Extirpando estos fragmentos cesaba el trastorno. No es de extrañar, pues, que sircay (cirujano) haya trepanado para curar la epilepsia. Es posible que hematomas subdurales o la epilepsia traumática, que ocasionaban dolor de cabeza, hayan inducido a trepanar el cráneo y a curar por medios racionales con una base empírica. Hoy es difícil establecer si curaban o no curaban a los pacientes; pero un interés primario es saber si intentaban curar. ${ }^{27,28}$

Méjico: de los aztecas la información más clara procede de los códices novohispanos, en especial el Libellus medicinalibus indorun herbis de Martin de la Cruz y Juan Badiano y el Códice Florentino de Fray Bernardino de Shagún. ${ }^{29}$ En el Libellus se menciona y se la consideró como una grave dolencia o debilidad del corazón y por ello recibió la denominación de yolpapatzmizquiliztli. Para la medicina azteca el corazón era el órgano central y en el residía el origen de las enfermedades. Sin embargo Viesca opina que en el Libellus puede encontrarse un intento por relacionar la epilepsia con el cerebro, al recomendar como uno de los remedios el cerebro cocido de comadreja y de zorra para transmitir las propiedades de esos animales a los epilépticos. Otro remedio animal fue el perro, el itzcuintli, de quien se recomendaba el empleo de su hiel. En la misma obra se aconsejó para la epilepsia el sahumerio de "buen olor" del nido de ratones quemado. En el manejo herbal una de las plantas recomendadas fue el tlatlacotic, el tlantlacuaye que era extensivo a otras parálisis y aquellos padecimientos que parten del costado y se extienden al corazón. Al mismo tiempo al considerar que la epilepsia era caliente, su tratamiento debía tender a disminuir el calor y para ello se recomendó el tlatlacotica o el acocoxpihuitl. ${ }^{30}$ En el estado de Chiapas, habita la etnia maya tzeltal constituida por unos 200.000 indígenas. ${ }^{31}$ Para ellos la epilepsia se conoce como 'tub tub ik al' que traduce "quien respira con agitación". ${ }^{22}$ Es una enfermedad adquirida la mayoría de las veces en la edad adulta y permanece en la sangre. No se considera un desorden del nacimiento ni existe asociación familiar, y por lo regular aparece en una persona de la familia. Los tzeltal le temen porque no tiene cura. ${ }^{19}$

Desde la concepción mágico-religiosa es una enfermedad mágica que está basada en el nagualismo, conjunto de tradiciones según las cuales el hombre a su nacimiento es asignado a un 'nahual' o alma animal que lo acompañará y compartirá su destino. De esta manera la enfermedad tiene origen en las luchas de los naguales de las fuerzas del bien y del mal, estos últimos enseñarán al alma de la persona secretos de hechicería y brujería, mientras los primeros trataran de evitarlo. Si el nagual compañero sobrevive a esta lucha y no muere, tendrá conocimientos de brujería y el individuo que presente crisis crónicas será considerado brujo. Aunque la enfermedad no se cura, se puede calmar con la administración de una bebida compuesta por semillas de dos hierbas conocidas como "kaxlam tunim" 
y "cheneh pox sbil", las cuales se toman a diario en agua fría durante una semana. ${ }^{22}$

Bolivia: los chipayas son un grupo étnico de origen uru que habita a $3.940 \mathrm{~m}$ de altitud sobre el nivel del mar, en una zona árida y salada del altiplano andino en la provincia Atahualpa, al norte del lago Coipasa, en el departamento de Oruro. ${ }^{32,33}$ La epilepsia se conoce con el nombre de tukuri. Los estudios etnográficos de Carod-Artal y Vásquez-Cabrera en $2004^{34,35}$, en las entrevistas realizadas a los yatri (sabio, curandero chipaya) encontraron concepciones como que el origen del tukuri es un mal de espíritu que entra por la nariz y la cabeza en forma de remolino de viento: "Entra por malicia y a veces se queda en el hueso, el músculo o a veces en la cabeza". ${ }^{36}$

Algunos tipos de crisis epilépticas fueron reconocidas en el mundo espiritual chipaya. El yatri describió crisis tonicoclónicas y atónicas, también oculógiras y el estado de amnesia poscrisis. Los yatris chipayas por lo regular establecen el pronóstico de las crisis: el peor ataque ocurre cuando la persona cae al piso como un muerto. También reconocen que en algunas ocasiones la persona puede morir por las crisis, las cuales se relacionan a veces con violencia doméstica o maltrato familiar, mientras otras son de origen mágico. Los yatris atribuyen el origen a varias causas, una de las cuales ocurre cuando el padre golpea fuerte a su hijo hasta hacerle perder la conciencia. Luego sufrirá crisis por una lesión cerebral ocurrida. También si se trabaja toda la noche hilando y se va a dormir a la madrugada un viento entra por la boca y la nariz, y se sufre tukuri porque se trabajó mucho. Lo que indica que los yatris identificaron la deprivación de sueño como precipitante de crisis. ${ }^{19}$

Existen diversas terapias naturales y mágicas para tratar el tukuri. Puede hacerse con curupancho "un insecto que viene en tiempos de lluvia", son hormigas con alas. Este se seca y se muele, se echa en una taza con agua hervida, luego se cuela y se toma en agua tibia los martes y viernes (de nuevo en días mágicos y ceremoniales) durante tres semanas. En tiempos de lluvia aparece también una mariposa llamada jesko, la traen los 'surazos', olas de frío polar procedentes del sur de Argentina que cambian en forma drástica el clima del altiplano. Se captura ese insecto, se seca, se hierve y se toma en infusión, los miércoles y viernes por la noche durante tres semanas. Un tercer tratamiento consiste en ingerir la infusión de un pájaro que aparece en tiempo de lluvia, llamado aspa, y que también llega con los surazos. Este pájaro debe tomarse hervido en su totalidad, incluidas las plumas. Otro tratamiento popular para tratar la epilepsia es tomar un pichón, cría de paloma se le corta la cabeza y se ingiere su sangre. En relación con el origen y tratamiento específico para las crisis en la infancia, el yatiri relata: "Los ataques en los niños se deben a que las mamás golpean a los niños, los golpean hasta caer y por ello tienen ataques. Para tratar y curarlos, la mamá tiene que pedir perdón. Si no pide perdón la mamá, el niño va a tener ataques. Existen dos formas de pedir perdón: si se tiene ganado, hay que hacer sacrificio ritual, una wilancha. Si no se tiene ganado, hay que llorar hasta pedir perdón varios días. Hombres y mujeres piden perdón de la misma manera". Los chipayas tienen noción de la influencia del factor genético. ${ }^{36}$

Brasil: Entre los indios kamayurá del alto Xingú, región triangular situada entre los ríos formadores occidentales del río xingú (von den Steinen, Ronuro y Batoví) y los dos orientales (Kuliseu y Kuluene), en el estado de Mato Grosso, a 800 kilómetros al nordeste de Brasilia $^{37}$, la doença o enfermedad de indio tiene su origen en la venganza del espíritu (mama'e) del animal muerto por el cazador o pescador. La epilepsia se conoce en kamayurá como teawurup, que significa enfermedad de tatú (armadillo), ya que se origina cuando el cazador lo mata con arco y flecha. El espíritu de este animal muerto atacará al hombre por la noche mientras duerme y le provocará una convulsión. Esta enfermedad afecta a niños, jóvenes y ancianos. Se caracteriza por episodios recurrentes con caídas al suelo, emisión de espuma por boca y sacudidas clónicas de extremidades. La enfermedad de tatú puede aparecer también en la infancia y su origen se debe a que el padre del niño afectado cazó a un tatú y el espíritu de éste atacó durante el sueño a su hijo. ${ }^{38}$

El peligro más serio de las crisis incontroladas o no tratadas es la muerte por ahogamiento, porque el trans- 
porte en el área kamayurá es en botes y la economía depende de la pesca. Los pajés kamayurá (chaman) practican el ritual pajelanca, una ceremonia que consiste en colocar las manos sobre el cuerpo y la cabeza del paciente, simultáneamente ora y fuma tabaco durante el ritual esparciendo el humo sobre el cuerpo del paciente epiléptico. Este ritual es realizado con fines terapéuticos. ${ }^{19}$ La doença de tatú se trata mediante la combinación de dos plantas, tsimó y wewurú, cuyas raíces se amasan y se diluyen en agua sin hervir; a continuación, se aplica en los ojos en forma de gotas, como norma a las cuatro horas de su preparación, durante tres días. Estas raíces también pueden administrarse por vía oral, hervidas, para inducir el vómito y evitar que se repitan las crisis; para ello se toman a diario cinco veces durante tres días. Otro tratamiento empleado es la infusión de enamuun, una raíz que se cuece y se ingiere cuatro veces al día (por la mañana, mediodía, tarde y a la hora de dormir) durante diez días. Wewurú y enamuun son las dos plantas más específicas para la enfermedad de tatú del adulto. Las crisis en los niños se tratan mediante la savia de una planta conocida como kamarapalap, que se aplica sobre la piel del niño y después sigue una serie de plegarias realizadas por el pajé cuando aplica la poción. ${ }^{38}$

Norteamérica: estudios etnobotánicos identificaron algunas plantas que fueron usadas para tratar las crisis epilépticas por nativos. ${ }^{39}$ Decocciones compuestas de cálamo, flor de mono y flor cardinal fueron usadas por las mujeres iroquois como emético para la epilepsia, mientras los hombres tomaban licopodio en decocciones compuestas como anticonvulsivante. ${ }^{40}$ La raíz pulverizada de pipa india fue dada a los niños cherokee. Los nativos de Delaware usaron infusión de algodoncillo para la epilepsia durante ciertas fases de la luna. Sin embargo la mayoría de estos estudios no proveen información de la enfermedad acerca del conocimiento, actitud y prácticas de los nativos norteamericanos. ${ }^{19}$

\section{Asia y Europa}

En la tradición ayurveda, el famoso médico hindú Sushruta en el siglo sexto antes de Cristo describió algunas formas clínicas de epilepsia. Le atribuyó causas psicofisiológicas a la epilepsia. La medicina tradicional china considera causas físicas como un viento o moco que bloquea el sistema cardíaco. ${ }^{41} \mathrm{En}$ la tradición judeo-cristiana, ha sido considerada como un castigo divino, por los pecados cometidos. El neurólogo francés Lhermitte estableció la indicación del exorcismo para casos genuinos de posesión demoníaca como diagnóstico diferencial de síndromes de origen no diabólico. En Europa antigua y en la edad media, además de la connotación mágica, la epilepsia fue referida como la enfermedad de la luna. ${ }^{23}$

\section{Discusión}

Los análisis transculturales en neurología ${ }^{42}$, suponen una vía de comprensión de las diferentes concepciones, tratamientos y efectos sociales que cada patología neurológica, en este caso la epilepsia, provoca en cada cultura. Aunque el pensamiento mágico sobre la epilepsia en las culturas indígenas ancestrales se ha visto modificado por la presencia del hombre blanco, coexistiendo en la actualidad con el tratamiento tradicional y científico occidental ${ }^{43}$, la base del éxito de cualquier estudio epidemiológico ${ }^{44}$ se centra en el conocimiento previo de la concepción. ${ }^{22}$ Es lo que se pretende con el presente trabajo al explorar el conocimiento actual y la concepción que de la epilepsia poseen los grupos indígenas alrededor del mundo. Para tal fin se realizó la búsqueda descrita con anterioridad y se encontraron estudios cualitativos tipo etnográficos y revisiones realizadas en poblaciones indígenas en países de los continentes americano y africano. Allí se describe la causa de la epilepsia concebida desde un pensamiento mágico en las tribus de los países de África, siendo el mal de ojo, el hechizo y la brujería fuentes de la enfermedad. También en algunas regiones de Tanzania tienen la creencia que existe afinidad por la luna apareciendo las crisis en la fase de luna llena.

En contraste, las etnias indígenas de los países de América han atribuido el origen de la enfermedad a diferentes fuentes, así por ejemplo los guaraníes en $\mathrm{Pa}-$ raguay la conciben como una entidad mágico-religiosa con un desequilibrio entre el alma sagrada y el alma animal provocado por una influencia maléfica. Por el contrario los pueblos incas del Perú usaron el sonko nanay para describir los eventos epilépticos y síncopes, 
siendo el sonko el corazón, mente y algunas veces el cerebro, y el nanay dolor o enfermedad quedando claro el concepto de tipo orgánico aunque la causa fue explicada a través de mitos o narraciones místicas. De la misma manera los aztecas en Méjico la consideraron como una grave dolencia del corazón y también hicieron intentos por relacionarla con el cerebro al recomendar uno de sus remedios el cerebro cocido de comadreja. De forma similar los mayas consideraron la epilepsia como una enfermedad adquirida que permanece en la sangre y no tiene asociación familiar. Aunque desde la concepción mágico-religiosa está basada en el nagualismo, tradición según la cual a cada hombre al nacer le es asignada el alma de un animal que lo acompañará y la epilepsia es el resultado de la lucha entre naguales. Por último los uru-chipayas en Bolivia le atribuyen la causa a un mal de espíritu que entra por la nariz y se queda en los huesos, músculo o en la cabeza. Sin embargo algunas crisis fueron relacionadas con violencia doméstica o maltrato y también identificaron la deprivación de sueño como precipitante de crisis.

Entre todos los uru-chipayas y los incas hicieron descripciones semiológicas bien definidas de las diferentes crisis epilépticas que están bien relacionadas con las clasificaciones actuales. Así mismo formularon el pronóstico de la enfermedad de acuerdo con el tipo de crisis.

A pesar de que no se estudió en forma específica el estigma social asociado con la epilepsia, en África, en la región del sur de Tanzania, las personas que no se someten al rito de curación, tal vez por la creencia en el contagio de la epilepsia, son discriminadas. No se encontró ninguna evidencia de los temores de contagio en la población nativa de América central y del sur. ${ }^{45-48}$ En general el tratamiento, además de los ritos mágicos, se basa en preparaciones con elementos extraídos de los tres reinos, aunque se encontró información acerca de trepanaciones realizadas en Perú.

\section{Conclusión}

La epilepsia ha sido reconocida como una entidad única por la mayoría de las culturas y sociedades. Con- siderada una de las enfermedades más temidas de la humanidad, desde tiempos antiguos se ha visto como su causa la posesión demoníaca o la venganza y el castigo por las fuerzas sobrenaturales ${ }^{23,49}$, lo que también ocurre en las poblaciones indígenas. Sin embargo, en la información obtenida en la presente revisión se resalta una fuerte concepción mágica lo que también ocurre en todas las tribus del territorio africano, mientras en América se encontraron diferencias entre los diferentes grupos, teniendo los incas en el Perú una medicina racional y con buenas calidades semiológicas, los síntomas fueron definidos, con similitudes entre sonko nanay y las nociones actuales sobre un episodio ictal. Los incas en contraste con otras culturas, no invocaron la posesión demoníaca como causa de la epilepsia ${ }^{26,50}$, a diferencia de otras como la maya y los uru-chipayas que le atribuyeron la causa basada en el nagualismo o a un mal de espíritu.

\section{Referencias}

1. Medina C. Epilepsia aspectos clínicos y psicosociales. Bogotá: Panamericana; 2004.

2. WHO. Epilepsy: an historical overview. Ginebra: World Health Organization; 2001 .

3. Satish J. Priority of Epilepsy Research in Asia. Epilepsia. 2005;46(Suppl 1):46-7.

4. Al-Adawi S, Al-Maskari M, Martin R, Al-Naamani A, Al-Riyamy K, Hussaini AA. Attitudes of Omani physicians to people with epilepsy. Neurosciences. 2000;5(1):18-21.

5. Caveness W, Gallup G. A survey of public attitudes towards epilepsy in 1979 with an indication of trends over the last thirty years. Epilepsia. 1980;21(5):509-18

6. Jacoby A, Wang W, Vu TD, Wu J, Snape D, Aydemir N, Parr J, et al. Meanings of epilepsy in its sociocultural context and implications for stigma: findings from ethnographic studies in local communities in China and Vietnam. Epilepsy Behav. 2008 Feb;12(2):286-97.

7. Choi-Kwon S, Park KA, Lee HJ, Park MS, Lee CH, Cheon SE et al. Familiarity with, knowledge of, and attitudes toward epilepsy in residents of Seoul, South Korea. Acta Neurol Scand. 2004; 110(1):39-45.

8. Mushi D, Hunter E, Mtuya C, Mshana G, Aris E, Walker R. Social-cultural aspects of epilepsy in Kilimanjaro Region, Tanzania: knowledge and experience among patients and carers. Epilepsy Behav. 2011 Feb; 20(2):338-43.

9. Dueñas J. Epilepsia: Historia y sociedad. Rev. Hosp. Psiquiátr. La Habana. 1999; 40(2): 171-77.

10. Devinsky O, Lai G. Spirituality and Religion in Epilepsy. Epilepsy Behav. 2008 May; 12(4):636-43.

11. Shorvon SD, Farmer PJ. Epilepsy in developing countries: a review of epidemiological, sociocultural, and treatment aspects Epilepsia. Epilepsia. 1988; 29 Suppl. 1:S36-54.

12. Gelfland M. Epilepsy in the African Central. Afr J Med. 1973;3:11-2.

13. Osuntokun B. Epilepsy in the African continent. In: Penry JK, editor. EpilepsyThe Eighth International Symposium. New York: Raven Press; 1977. p. 365-78.

14. Hamdi H, Al-Husaini A, Al-Hadithi F. The epilepsies: clinical and epidemiological aspects/availability and desirability of services. In: Penry JK, editor. Epi- 
lepsy: The Eighth Internarional Symposium. New York: Raven Press; 1977. p. 393-9.

15. Virmani V, Kaul V, Juneja J. Sociocultural and economic implications Nof epilepsy in India. In: Penry JK, editor. Epilepsy: The Eighth International Symposium. New York: Raven Press; 1977. p. 385-92

16. Estrella E. Medicina aborígen: la práctica médica aborigen de la Sierra Ecuatoriana. Quito, Ecuador : Epoca; 1977.

17. Jilek-Aall L, Jilek W, Miller J. Clinical and genetic aspects of seizure disorders prevalent in an isolated African population. Epilepsia. 1979 Dec; 20(6):613-22

18. Arawatife A, Longe A, Arawatife M. Epilepsy and psychosis: a comparison of societal attitudes. Epilepsia. 1985; 26:1-9.

19. Carod F, Vázquez C. An Anthropological study about epilepsy in native tribes from Central and South America. Epilepsia. 2007;48(5):886-93.

20. Gerrits C. Epilepsy care in a non-cinical setting. A medical-anthropological study among the Bassa and the Kpelle in the rainforest of Liberia, west Africa. Trop Geogr Med. 1994; 46(suplemento):S13-7.

21. Ebner E. The history of the Wangoni and their origin in the South African Bantu tribes. Peramiho [Tanzania]: Benedictine Publications Ndanda; 1987.

22. Carod FJ, Vázquez-Cabrera C. Pensamiento mágico y epilepsia en la medicina tradicional indígena. Rev Neurol. 1998; 26(154):1064-8.

23. Jilek-Aall L. Morbus Sacer in Africa: Some religious aspects of epilepsy in traditional cultures. Epilepsia. 1999;40(3):382-6.

24. Vara A. Corrientes en el mundo guaranítico: todo es historia. Buenos Aires: Alemann SRL; 1985.

25. González Torres DM. Catálogo de plantas medicinales y alimentarias y útiles usadas en Paraguay. Asunción: Comuneros; 1981

26. Burneo JG. Sonko-Nanay and epilepsy among the Incas. Epilepsy Behav. 2003 Apr; 4(2):181-4.

27. Cruz-Campos G. Concepción y evolución histórica de la epilepsia en el Perú precolombino y del Virreinato. Rev Neurol. 1998;27(159):862-66.

28. Pomata H, Campos M. Epilepsy in pre-Columbian times. Childs Nerv Syst. 2008;24:671-3.

29. De la Cruz M JB, Sahagun Bd. Libellus medicin alibus in dorum herbis y Códice Florentino. México : Instituto Mexicano del seguro social, Editorial Giunti Barberá; 1979.

30. Rodríguez-Sala ML, Cuevas NS. En torno a un tratamiento de la epilepsia en la Nueva España: el uso del cráneo humano por tres médicos en Puebla, 1702. Rev Mex Neuroci. 2012; 13(5): 286-93.

31. De Vos J. Vivir en frontera: la experiencia de los indios de Chiapas. México: Centro de Investigaciones y Estudios Superiores en Antropología Social; 1994.

32. Barrientos IF. Chipaya, reliquia viviente : estudio monográfico. Chipaya (Oruro, Bolivia): [s.n.]; 1990.
33. Delgadillo J. La nación de los urus: Chipaya 1984. Serie Nosotros. Bolivia: Centro Diocesano de Pastoral social; 1998.

34. Carod-Artal F, Vázquez-Cabrera C. Paleopatología neurológica en las culturas precolombinas de la costa y el altiplano andino (I). Deformaciones craneales intencionales. Rev Neurol. 2004;38:791-7.

35. Carod-Artal FJ, Vázquez-Cabrera CB. Paleopatología neurológica en las culturas precolombinas de la costa y el altiplano andino (II). Historia de las trepanaciones craneales. Rev Neurol. 2004;38:886-94.

36. Carod-Artal FJ, Vázquez-Cabrera CB. Estudio etnográfico sobre las enfermedades neurológicas y mentales entre los urus-chipayas del altiplano andino. Rev Neurol. 2005;41(2):115-25.

37. Samain E. De um caminho para o outro; mitos e aspectos da realidade social nos índios Kamayurá (Alto Xingú). Rio de Janeiro: Museu Nacional; 1980.

38. Carod-Artal FJ, Vázquez-Cabrera CB. Antropología neurológica entre los indios Kamayurá del Alto Xingú. Rev Neurol. 2001;32(7):688-95.

39. Turner NJ, Royal British Columbia M. Thompson ethnobotany : knowledge and usage of plants by the Thompson Indians of British Columbia. Victoria: Royal British Columbia Museum; 1990

40. Herrick J. Iroquois medical botany. Albany: State University of New York; 1977.

41. Kleinmann A, Wang W, Li S. The social course of epilepsy: chronic illness as social experience in interior China. Soc Sci Med. 1995;40(10):1319-30.

42. Carod F, Cabrera CV. Una visión transcultural de la patología neurológica y mental en una comunidad maya tzeltal de los Altos de Chiapas. Rev Neurol. 1996;24:848-54.

43. Janzen J. Ideologies and institutions in the precolonial history of equatorial Africa therapeutic systems. Soc Sci Med. 1979;13-B:317-26.

44. Rwiza H, Kilonzo G, Haule J, Matuja W, Mteza I, Mbena P. Prevalence and incidence of epilepsy in Ulanga, a rural Tanzanian district: a community-based study. Epilepsia. 1992;33:1051-6.

45. Nyame P, Biritwum R. Epilepsy: knowledge, attitude and practice in literate urban population, Accra, Ghana. West Afr J Med. 1997 Jul-Sep; 16(3):139-45

46. Arborio S, Dozon J. Sociocultural dimension of epilepsy (kirikimasien) in a Bambara rural area (Mali). Bull Soc Pathol Exot. 2000 Nov; 93(4):241-6.

47. Millogo A, Ratsimbazafy V, Nubukpo P, Barro S, Zongo I, Preux P. Epilepsy and traditional medicine in Bobo-Dioulasso (Burkina Faso). Acta Neurol Scand. 2004 Apr; 109(4):250-4

48. Baskind R, Birbeck G. Epilepsy care in Zambia: a study of traditional healers. Epilepsia. 2005;46:1121-6.

49. Riggs AJ, Riggs JE. Epilepsy's role in the historical differentiation of religion, magic, and science. Epilepsia. 2005;46(3):452-3.

50. Mendizábal J. The ritual of the Bacabs: epilepsy among the Mayans. Neurology. 2001;suppl 3:A93. 